\title{
Caracterización espacial de la frecuencia e intensidad de tormentas desde el satélite GOES-12 y la Estación Meteorológica del Observatorio de Huancayo
}

\author{
Spatial characterization of storm's frequency and intensity from the \\ GOES-12 satellite and the meteorological station from Huancayo \\ Observatory
}

Jacinto Arroyo Aliaga', Elizabeth Machuca Manrique², Pedro Gurmendi Párraga ${ }^{3}$ Universidad Continental

\section{RESUMEN}

Objetivos: Caracterizar la frecuencia e intensidad de las tormentas de precipitaciones a partir de imágenes obtenidas del Satélite Geoestacionario Operacional Ambiental (Geostationary Operational Environmental Satellite, GOES-12) y de datos registrados en la Estación Meteorológica del Observatorio de Huancayo, durante los ciclos hidrológicos que comprende entre julio de 2012 a junio de 2014. Métodos: Se utilizó el método del hidroestimador que georreferencia el punto y la intensidad mediante la relación empírica exponencial entre la precipitación estimada por radar y la temperatura de brillo del tope de las nubes a partir del canal infrarrojo que produce tasas de precipitación en tiempo real y el método de intensidad de precipitación que evalúa el índice de intensidad en milímetros por hora; sin embargo, debido a que la información que se registra en el pluviómetro tiene sesgos por efectos de contorno, se tomó como recurso el análisis de tormentas en 120 minutos con lluvia máxima en 12 horas. Resultados: Se caracterizó las precipitaciones en ligeras ( 11 eventos), moderadas (8 eventos), fuertes ( 3 eventos) y extremas ( 5 eventos) a partir del análisis de los datos físicos de la estación y se construyeron mapas de intensidades pluviométricas acopladas en tiempo-espacio de dos eventos extremos, además se presenta los puntos de conexión entre la precipitación, temperatura y presión atmosférica. Conclusiones: Se identificó los puntos y el área de impacto de las precipitaciones y su correlación con los datos de precipitación de la Estación Meteorológica del Observatorio de Huancayo.

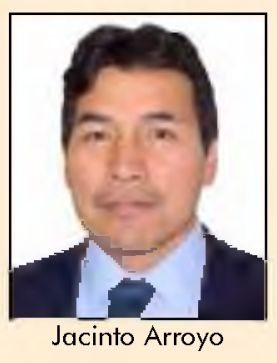

jarroyox@gmail.com

Historial del artículo: Recibido: 15 de junio de 2014 Aprobado: 26 de junio de 2014 Disponible en línea: 30 de junio de 2014

Palabras claves: Tormenta, frecuencia, intensidad, precipitación, satélite GOES-12, Huancayo. 


\section{ABSTRACT}

Objectives: To characterize the rain storms' frequency and intensity through obtained images from the Geostationary Operational Environmental Satellite (GOES-12) and recorded data at the meteorological station from Huancayo Observatory, during hydrological cycles comprising between july 2012 to june 2014. Methods: It was used the hydro-estimator method which georeference the point and intensity by the exponential empirical relationship between radar estimated precipitation and the cloud tops brightness temperature through the infrared canal which produces precipitation rates in real time and the precipitation intensity method which assesses the intensity rate in millimeters per hour; however, because of the information is recorded in the pluviometer presents biases by boundary effects, we took as a resource the storms analysis in 120 minutes with maximum rain in 12 hours. Results: Rainfall was classified as light (1 1 events), moderate ( 8 events), strong ( 3 events) and extreme (5 events) according to the physical data analysis of the station and rainfall intensities maps were constructed coupled in timespace about two extreme events, also the connection points were presented between precipitation, temperature and atmospheric pressure as well as the satellite images during the extreme storms development. Conclusions: Points and rainfall impact area were identified, also its correlation with precipitation data from the meteorological station from Huancayo Observatory.

Keywords: Storm, frequency, intensity, precipitation, GOES-12 satellite, Huancayo.

\section{INTRODUCCIÓN}

El contexto actual de cambio climático exige ampliar el conocimiento sobre las variaciones espaciales y temporales de las precipitaciones extremas, asociadas a un aumento de la frecuencia e intensidad de las tormentas de lluvias (1). Las tormentas generan adversidades de alto impacto socioeconómico en la sociedad, porque las variaciones de frecuencia e intensidad en 24 horas están favoreciendo procesos geomorfológicos dinámicos que provocan remociones de masa (2).

Las tormentas son fenómenos convectivos que producen precipitaciones intensas de gran intensidad, baja frecuencia temporal y aparente distribución espacial (3). Por su formación son procesos hidrometeorológicos que provocan peligros naturales del tipo geomorfológico, como procesos de erosión superficial, movimientos de masa, inundaciones fluviales, arroyamiento torrencial, y cambio en los causes y en las llanuras aluviales, que desencadenan desastres, afectando a la población, vivienda e infraestructura (4).

Las tormentas presentan una estructura que se compone de uno o varios centros activos denominados células. Una célula es una nube elemental que comprende en algún momento de su desarrollo como mínimo una corriente ascendente bien establecida y una descendente (5). El ciclo de vida de una tormenta oscila entre media hora y varias horas. Durante ese tiempo la tormenta puede permanecer estacionaria o recorrer de 15 a $30 \mathrm{~km}$, en promedio, en la dirección de los vientos dominantes (6).

Las nuevas teorías modernas sobre tormentas señalan que las depresiones de altura siempre tienen un reflejo en niveles bajos y superficie, que se manifiesta en algún tipo de anomalía (presión, temperatura, estabilidad, viento) y estos pueden producir precipitaciones en las épocas del otoño, primavera que coincide con las primeras irrupciones de masas de aire frío en los altos niveles de la atmósfera hacía las latitudes medias (7).

Algunas investigaciones realizadas en el Valle del Mantaro y en el Perú, han analizado la variabilidad y cambio climático regional. Pero sus objetivos estaban centrados a la distribución de las precipitaciones por sus repercusiones en el manejo de los recursos naturales y la gestión del riesgo, así como el desarrollo económico y social (8). Sin embargo, estudios de tormentas 
hidrometeorológicas no han sido muy estudiados por sus procesos complejos y aleatorios (9).

Debido a la inexistencia de observaciones de tormentas en el Perú por la carencia de redes conectadas se han planteado las siguientes interrogantes: ¿Cuál es el comportamientode la frecuenciaeintensidad de las tormentas de precipitaciones usando metodologías que corresponden a interpretaciones por satélite?, y ¿̇Cómo se manifiesta las precipitaciones intensas a partir de las imágenes obtenidas del satélite GOES-12?

El uso de herramientas sofisticadas de geoprocesamiento e interpretación de imágenes satelitales, permitió determinar la distribución, la frecuencia e intensidad de las tormentas para la zona de estudio. Es la razón que la investigación tuvo como objetivo en primera instancia caracterizar la frecuencia e intensidad de las tormentas de precipitaciones y cómo se manifiestan las precipitaciones intensas a partir de imágenes obtenidas del satélite GOES-12; luego se validó con datos registrados en la Estación Meteorológica del Observatorio de Huancayo, determinando la serie de precipitaciones intensas totales no menor a $20 \mathrm{~mm}$, consideradas como extremas según su clasificación (10). Se utilizó el software ENVI, para combinar las bandas de imágenes de satélite y el ArcGIS para georreferenciar su distribución e intensidad (11).

\section{MATERIAL Y MÉTODOS}

Se trabajó con el método del hidroestimador a partir de imágenes satelitales GOES-12 y del análisis de las variables meteorológicas de la Estación Meteorológica del Obsevatorio de Huancayo. Se utilizó la base de datos horarios, diarios y mensuales de los años 2012-2013 compuesta por 365 registros anuales y 48 mensuales de las variables de presión atmosférica, temperatura, y precipitación (12).

El hidroestimador es un método enteramente automático que utiliza una relación empírica exponencial entre la precipitación (estimada) y la temperatura de brillo del tope de las nubes (extraída del canal infrarrojo del satélite GOES-12, produciendo tasas de precipitación en tiempo real; a través de la tendencia de temperatura de la nube y de informaciones sobre la textura. Luego se realiza un ajuste de área cubierta por la precipitación por el uso de un software de GIS (13).

El hidroestimador fue desarrollado a fin de resolver problemas antes generados por el auto-estimador, que consistía en utilizar una curva de tendencia de temperatura y no la información de textura (asumiendo precipitación cero para píxeles en la región de baja variación espacial debajo de la posición en la que se encuentran los cirros). Así, el hidroestimador utiliza diferentes discriminaciones para el cálculo de áreas de lluvia y no lluvia y nuevos ajustes para el efecto de la humedad disponible (14).

El modelo de estimación implementado es una adaptación de aquél utilizado por la NESDIS (15). Este modelo pasó por varias modificaciones y para ser implementado operacionalmente en la División de Sistemas y Satélites Ambientales (DSA) fue enteramente adaptado para las condiciones y estructura de la precipitación sobre América del Sur (16). Los productos obtenidos de precipitación exhiben la precipitación estimada en la imagen GOES más reciente recibida por la DSA y la precipitación acumulada durante el período entre las 12:00 horas del día anterior y las 12:00 horas del día actual. La ausencia de productos en una determinada hora o en un determinado día es consecuencia de la falta de imágenes GOES o de datos de viento producidos por el modelo del CPTEC (17).

\section{Delimitación espacial de la investigación}

El trabajo se limita a las provincias de Chupaca y Huancayo, que se encuentran en la región Junín, como se muestra en la figura 1 .

La clasificación de tormentas se ha realizado sobre la base de los valores de intensidad 


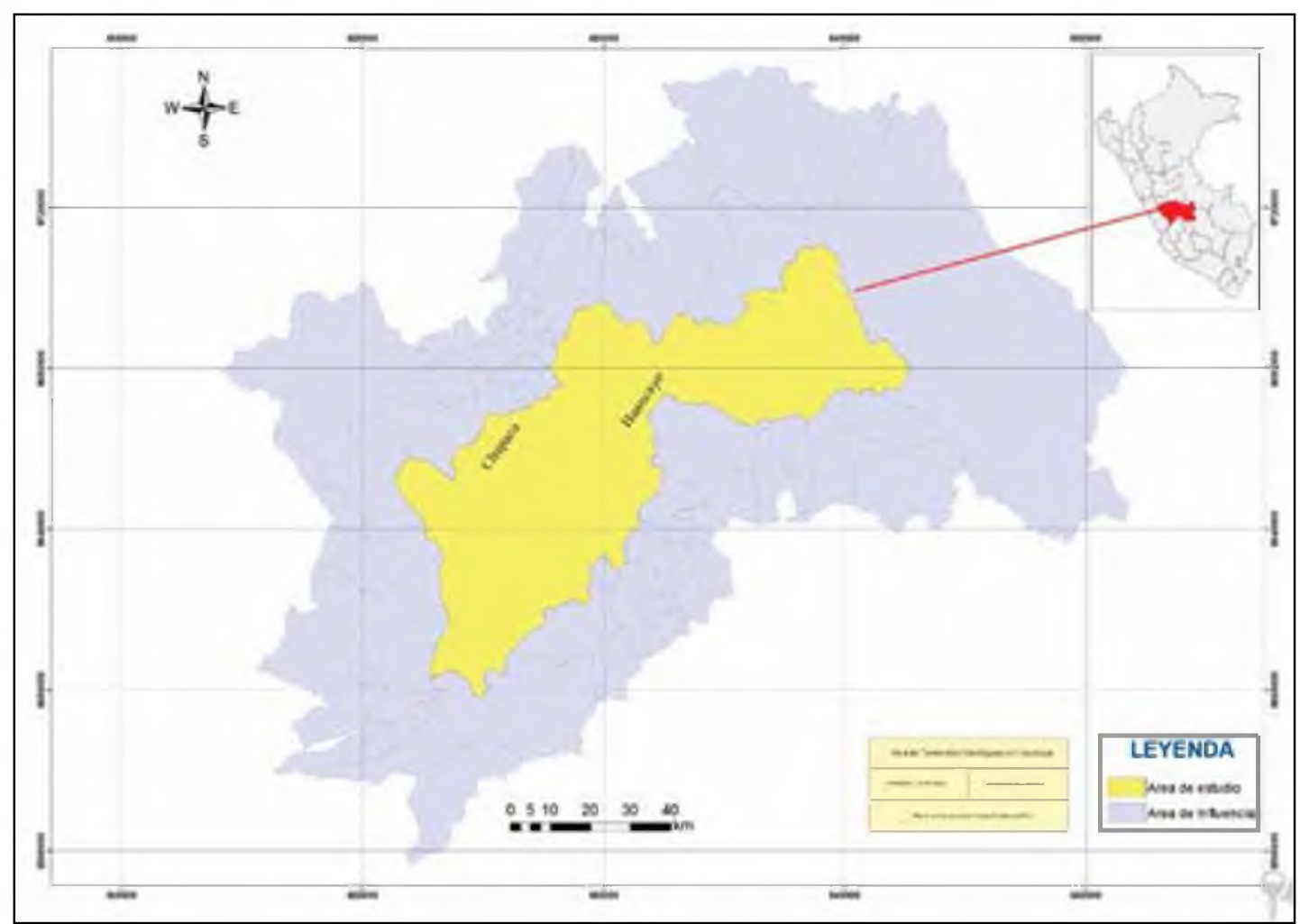

Figura $\mathrm{N}^{\circ}$ 1: Ubicación geográfica de la investigación.

$(\mathrm{mm} / \mathrm{h})$ que se muestra en la tabla 1. Los criterios también son descritos para lograr una clasificación normal.

Para la caracterización de la precipitación se estandarizó los datos al promedio de la altura del agua precipitada en un día de ocurrencia. Para conocer este índice se

Tabla $N^{\circ}$ 1: Clasificación de tormentas. aplica la siguiente fórmula (18):

$$
C p=\frac{P}{D p}
$$

Donde:

\begin{tabular}{|c|c|c|}
\hline Intensidad & $\mathrm{mm} / \mathrm{h}$ & Criterios \\
\hline Ligera & $(5-10)$ & $\begin{array}{l}\text { Las precipitaciones son intensas en forma de Chubasco. El acumulado en lluvia no } \\
\text { debe ser menos a } 5 \mathrm{~mm} \text { en una hora, pero el total no debe superar a los } 10 \mathrm{~mm} \text {. }\end{array}$ \\
\hline Moderada & $(10-15)$ & $\begin{array}{l}\text { Las precipitaciones son intensas en forma de chaparrones o chubasco. El acumulado } \\
\text { en lluvia no debe ser menos a } 10 \mathrm{~mm} \text { en una hora y el total no debe ser mayor a } \\
15 \mathrm{~mm} \text {. Excepcionalmente pueden darse precipitaciones menores a } 10 \mathrm{~mm} \text { pero no } \\
\text { debe exceder los } 15 \mathrm{~mm} \text {. }\end{array}$ \\
\hline Fuerte & $(15-20)$ & $\begin{array}{l}\text { Las precipitaciones son intensas en forma de chaparrones o chubasco, acompañado } \\
\text { de granizada y rayos permanentes. El acumulado en lluvia no debe ser menos a } 10 \\
\mathrm{~mm} \text { en una hora, pero el total no debe ser menor a } 15 \mathrm{~mm} \text { ni superar los } 20 \mathrm{~mm} \text {. } \\
\text { Excepcionalmente el acumulado de una hora puede ser de } 5 \mathrm{~mm} \text {. }\end{array}$ \\
\hline Extremo & $(20-25>$ & $\begin{array}{l}\text { Las precipitaciones son intensas en forma de chaparrones o chubasco, acompañado } \\
\text { de granizada, pedrisco y rayos permanentes. El acumulado en lluvia no debe ser } \\
\text { menos a } 15 \mathrm{~mm} \text { por hora, pero el total no menor a } 20 \mathrm{~mm} \text { aunque sí puede superar } \\
\text { los } 30 \mathrm{~mm} \text {. Generalmente el acumulado de una hora excede los } 20 \mathrm{~mm} \text {. }\end{array}$ \\
\hline
\end{tabular}

Fuente: Elaboración para la zona de estudio a partir de la información del Centro de Pronóstico del Tiempo y Estudios Climáticos (CPTEC). 
$C_{p}=$ Concentración de lluvia en mm/día. $\mathrm{P}=$ Precipitación en $\mathrm{mm}$.

$\mathrm{Dp}=$ Promedio de días con lluvia apreciable

Este promedio de días con lluvia apreciable se obtuvo primero para la concentración diaria y mensual. El procedimiento consistió en sumar todas las horas de precipitación de estos días de tormentas. Para el análisis de la precipitación de lluvias intensas se evaluó índice de intensidad de precipitación que se mide en milímetros por hora; sin embargo, como la información que se registra en el pluviógrafo no es lo suficientemente confiable, se tomó como recurso la lluvia máxima en 12 horas (19).

Para el análisis de la intensidad-duraciónperíodo de retorno se utilizó la fórmula de Chen (20). Útil para estimaciones dentro del intervalo de 5 minutos a 24 horas. Para la aplicación de la fórmula de Chen se requiere conocer los cocientes:

1) Lámina de lluvia-duración, $R=\frac{R^{\top}{ }_{1}}{R_{24}^{\top}}$

donde $\mathrm{R}^{\mathrm{T}}$, es la precipitación acumulada en una hora y período de retorno $T$ y $R^{T}{ }_{24}$ es la precipitación acumulada en 24 horas y período de retorno $T$ (21).

La fórmula propuesta por Chen para estimar la lluvia en milímetros de duración (t) en minutos y el periodo de retorno $(T)$ en años, es:

$R T \dagger=\frac{a R^{2} \log \left(10^{(2-x)} T^{(x-1)}\right)}{(t+b)^{\dagger}}$

Esta es válida para las siguientes condiciones: $T$ e $\bullet 1$ año y $5 \mathrm{~min}<t<24$ horas. Donde $\mathrm{R}^{2}{ }_{1}$ es la lluvia registrada para una duración de una hora y período de retorno de 2 años, en milímetros; a y b son parámetros en función del cociente R (22).

\section{RESULTADOS}

La frecuencia de eventos que se presentaron en 24 meses de análisis de tormentas, se muestran en la tabla 2 y figura 2 , donde se observa la frecuencia de tormentas ligeras, moderadas, fuertes y extremas. Las precipitaciones extremas tuvieron 5 eventos superiores a $20 \mathrm{~mm}$ equivalente a $\left(20 \mathrm{l} / \mathrm{m}^{2}\right)$ en la primera hora. Pero las tormentas ligeras fueron las que tuvieron

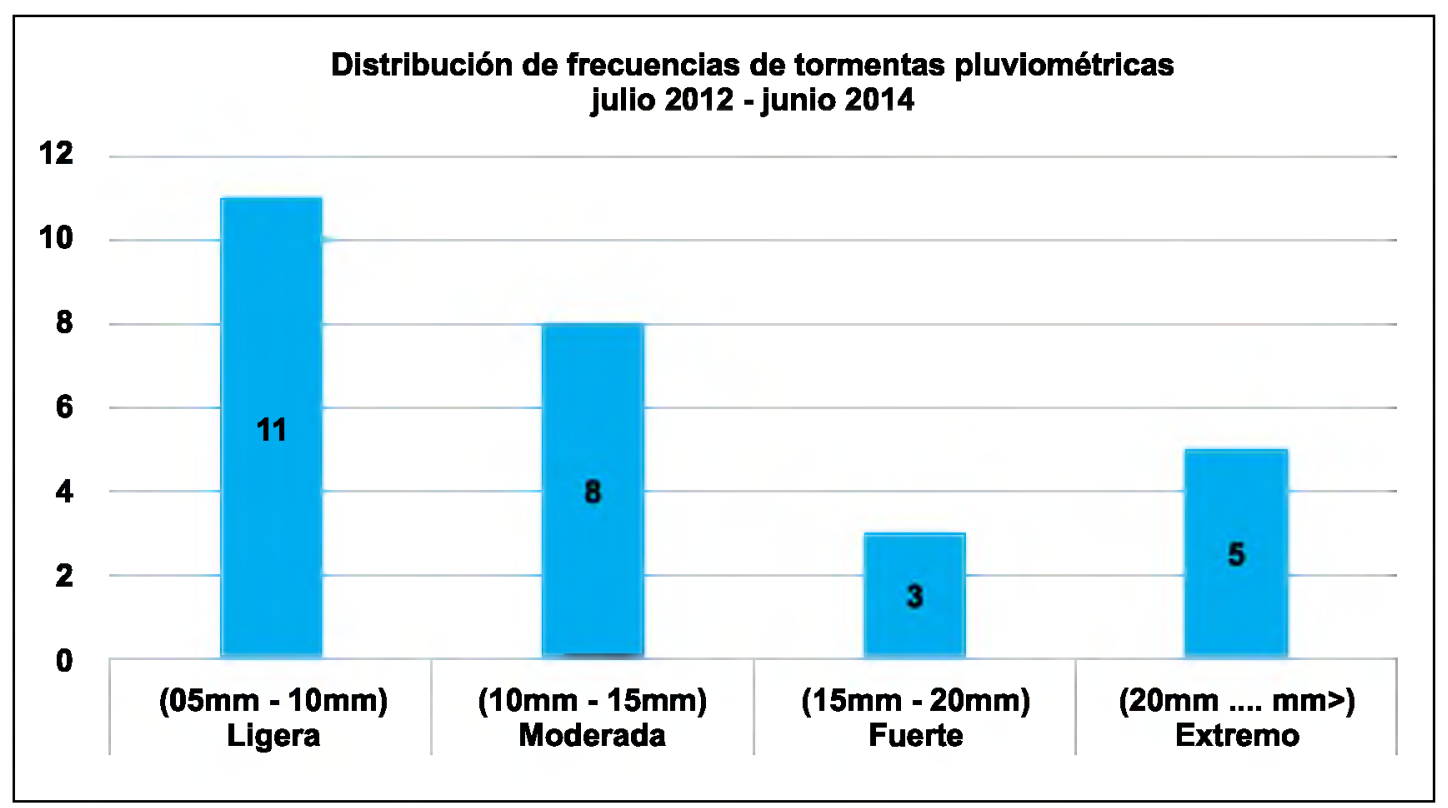

Figura $\mathrm{N}^{\circ}$ 2: Distribución de frecuencias de tormentas pluviométricas en 24 meses durante el período de investigación: julio 2012 - junio 2014. 
Tabla N ${ }^{\circ}$ 2: Distribución de frecuencias de tormentas registrados en la Estación Meteorológica del Observatorio de Huancayo.

\begin{tabular}{cccc}
\hline Nro & Intensidad & Rango & Frecuencia \\
\hline 1 & Ligera & {$[05 \mathrm{~mm}-10 \mathrm{~mm}]$} & 11 \\
2 & Moderada & {$[10 \mathrm{~mm}-15 \mathrm{~mm}]$} & 8 \\
3 & Fuerte & {$[15 \mathrm{~mm}-20 \mathrm{~mm}]$} & 3 \\
4 & Extremo & {$[20 \mathrm{~mm}-\ldots . . \mathrm{mm}>$} & 5 \\
\hline
\end{tabular}

11 frecuencias durante el estudio, seguido de tormentas moderadas con ocho eventos y fuertes con tres eventos. El total fue de 27 tormentas registradas.

En la tabla 3 se muestran la distribución de frecuencias, la fecha y la intensidad de las precipitaciones en la primera y segunda hora. El total supera los $5 \mathrm{~mm}$ de precipitación pero no excede los $10 \mathrm{~mm}$. Generalmente la mayor concentración de la intensidad corresponde a la primera hora. La distribución espacial, la frecuencia

Tabla $N^{\circ}$ 3: Distribución de frecuencias de tormentas ligeras registradas en la Estación Meteorológica del Observatorio de Huancayo.

\begin{tabular}{ccccc}
\hline Nro. & Fecha $y$ hora de inicio & 1ra. hora & 2da. hora & Total \\
\hline 1 & $22 / 08 / 201317: 10-17: 50$ & 5,08 & & 5,08 \\
2 & $03 / 06 / 201316: 00-16: 20$ & 6,60 & & 6,60 \\
3 & $11 / 06 / 201317: 50-18: 20$ & 7,37 & & 7,37 \\
4 & $08 / 04 / 201318: 00-18: 50$ & 5,08 & 0,25 & 5,33 \\
5 & $08 / 03 / 201316: 40-17: 30$ & 5,84 & 1,52 & 7,37 \\
6 & $04 / 03 / 201316: 10-16: 30$ & 6,10 & 0,00 & 6,10 \\
7 & $11 / 02 / 201317: 50-18: 30$ & 6,35 & 0,00 & 6,35 \\
8 & $27 / 01 / 201319: 00-19: 50$ & 5,84 & 2,03 & 7,87 \\
9 & $23 / 01 / 201316: 40-17: 30$ & 5,33 & 1,78 & 7,11 \\
10 & $18 / 11 / 201217: 00-17: 50$ & 6,10 & 2,29 & 8,38 \\
11 & $20 / 09 / 201218: 00-18: 50$ & 5,59 & 0,51 & 6,10 \\
\hline
\end{tabular}

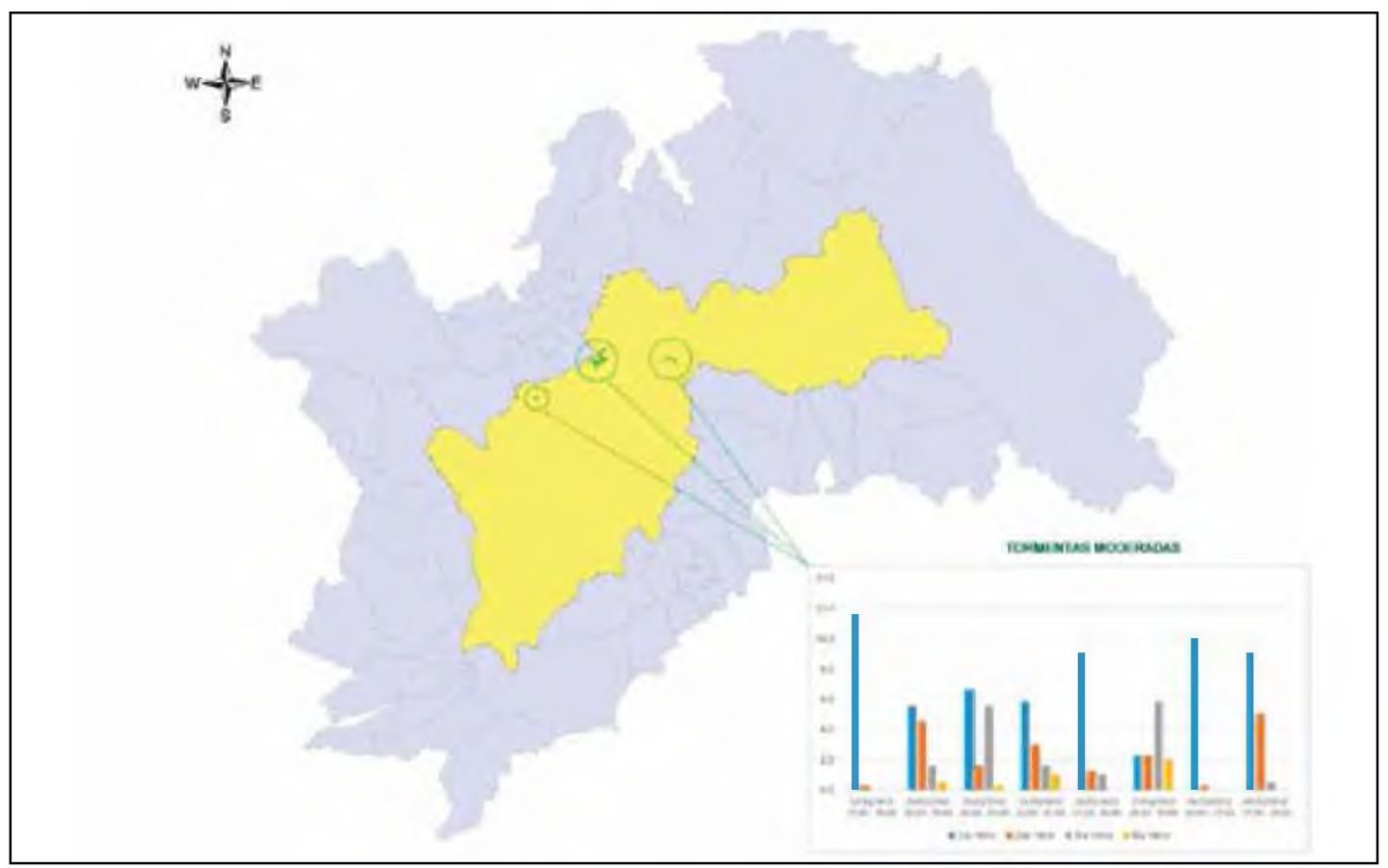

Figura $\mathrm{N}^{\circ}$ 3: Distribución espacial de lluvias moderadas en la zona de impacto. 


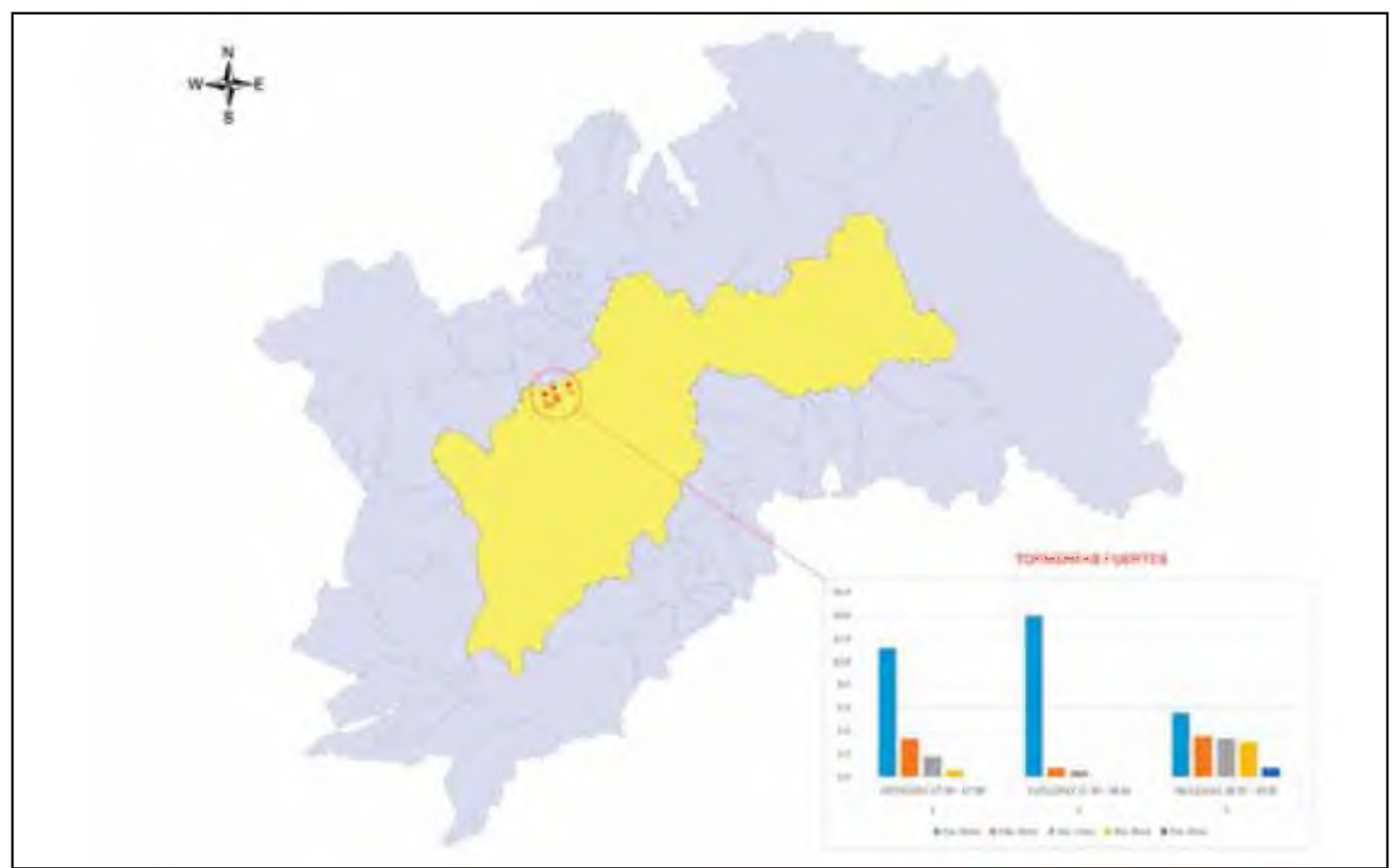

Figura $\mathrm{N}^{\circ}$ 4: Distribución espacial de lluvias fuertes en la zona de estudio.

e intensidad de tormentas moderadas extraídas a partir de las observaciones del satélite GOES 12 se presentan en la figura 3 , donde se muestran 8 eventos que superan los $10 \mathrm{~mm}$ de precipitación en el acumulado pero no exceden los $15 \mathrm{~mm}$. Además las mayores concentraciones se encuentran en los bordes de la delimitación. En la figura 4, se presenta la distribución espacial, la frecuencia e intensidad de las tormentas fuertes, procesadas a partir de las imágenes del satélite GOES-12. Las tres precipitaciones excedieron los $15 \mathrm{~mm}$ en el acumulado, pero no fueron superiores

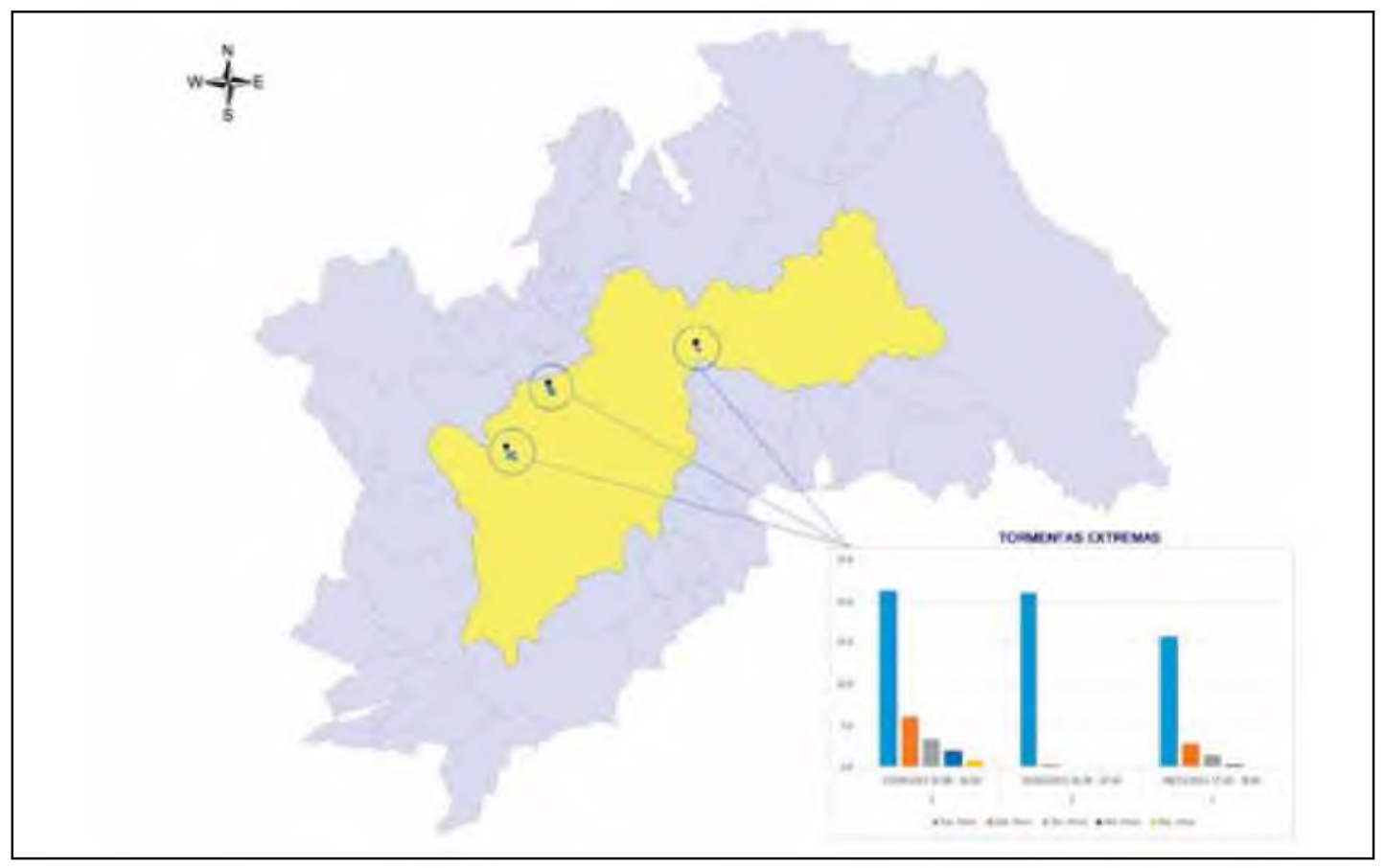

Figura $\mathrm{N}^{\circ}$ 5: Distribución de tormentas extremas que corresponde desde julio 2012 - diciembre 2013. 
a los $20 \mathrm{~mm}$ de precipitación. Las tres precipitaciones fueron localizadas en la provincia de Chupaca, en la frontera con el distrito de Sicaya.

Para presentar el tratamiento del análisis de tormentas extremas, se ha recurrido a dividir en dos partes. Primero georreferenciar su distribución espacial, su frecuencia e intensidad desde julio hasta diciembre del 2013, con la finalidad de tener una caracterización espacial, figura 5. Luego se presentan en las figuras 6 y 7 , dos formas de indicar la intensidad a detalle. Las
Además, la figura 6 muestra el comportamiento de la tormenta del día 25 de abril de 2014, que tuvo una precipitación acumulada neta de $61 \mathrm{~mm}$ en dos horas. Pero la primera hora fue de $49 \mathrm{~mm}$, precipitación que se puede considerar atípica y de solo un núcleo de concentración. Mientras que la figura 7 , muestra la tormenta del día 4 de mayo de 2014, con tres núcleos de concentración superando $68 \mathrm{~mm}$. Esta precipitación también se considera extrema y atípica. Ambos eventos descritos solo tuvieron una hora de máxima intensidad.

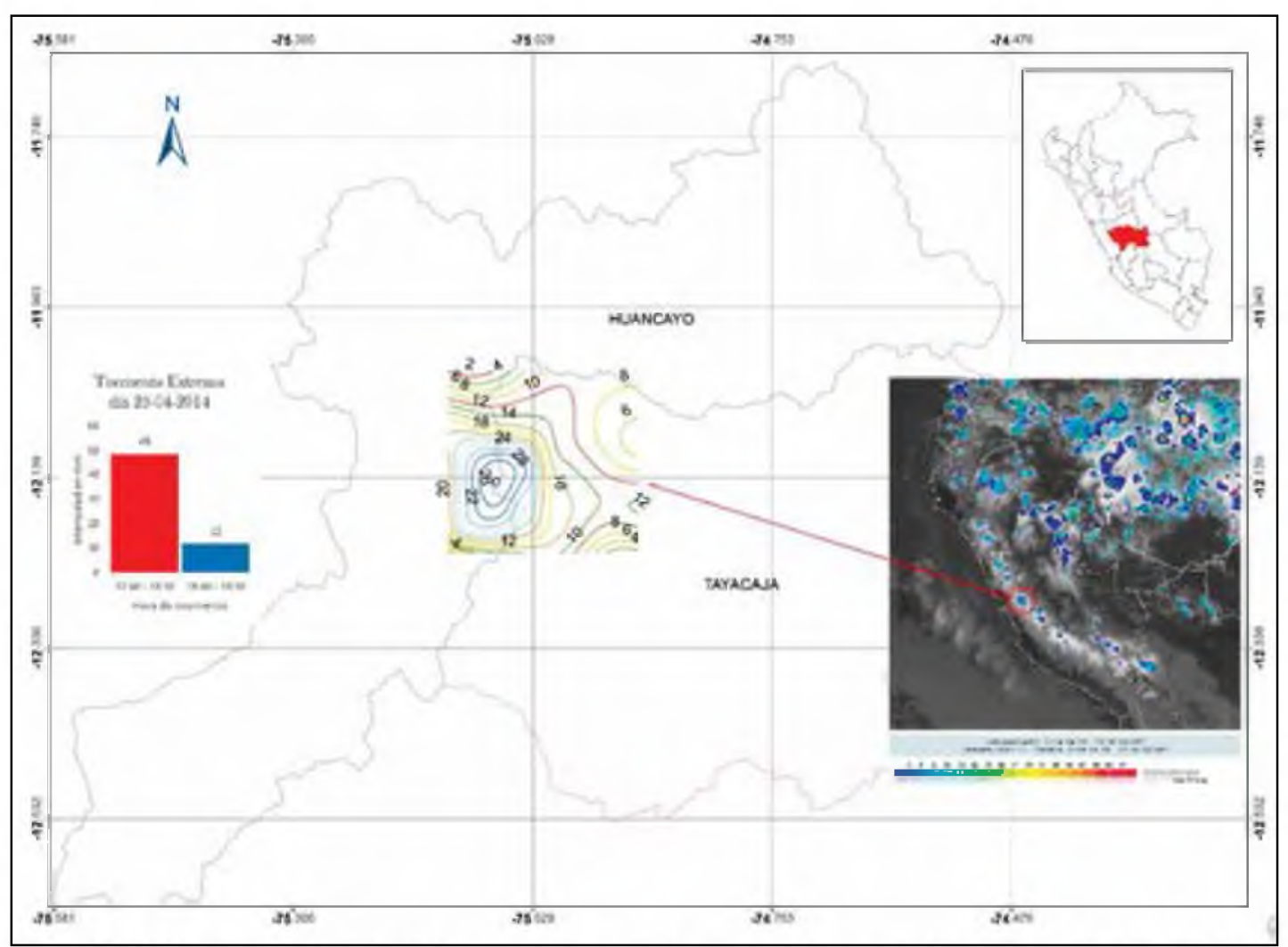

Figura $\mathrm{N}^{\circ}$ 6: Análisis espacial y de tormenta de precipitaciones extremas del día 25 de abril de 2014.

isoyetas caracterizan la distribución espacial y la concentración de precipitaciones en $\mathrm{mm}$. En la misma figura se observa una superposición de las imágenes satelitales GOES-12, que muestra el movimiento general de las tormentas que se producen como producto del movimiento de las masas. La intensidad se presenta en una figura de barras que por su coloración indican la cantidad de precipitación en las zonas de impacto.
La caracterización del comportamiento de las variables de temperatura, presión atmosférica y precipitación de los eventos extremos se observa en la figura 8. Las tormentas muestran características comunes, se puede describir en un aumento inicial de la temperatura durante la mañana para luego descender precipitosamente en el mismo instante en que se presentan las tormentas. La presión atmosférica tiene un comportamiento inverso debido a la incursión misma de una masa de aire fría 


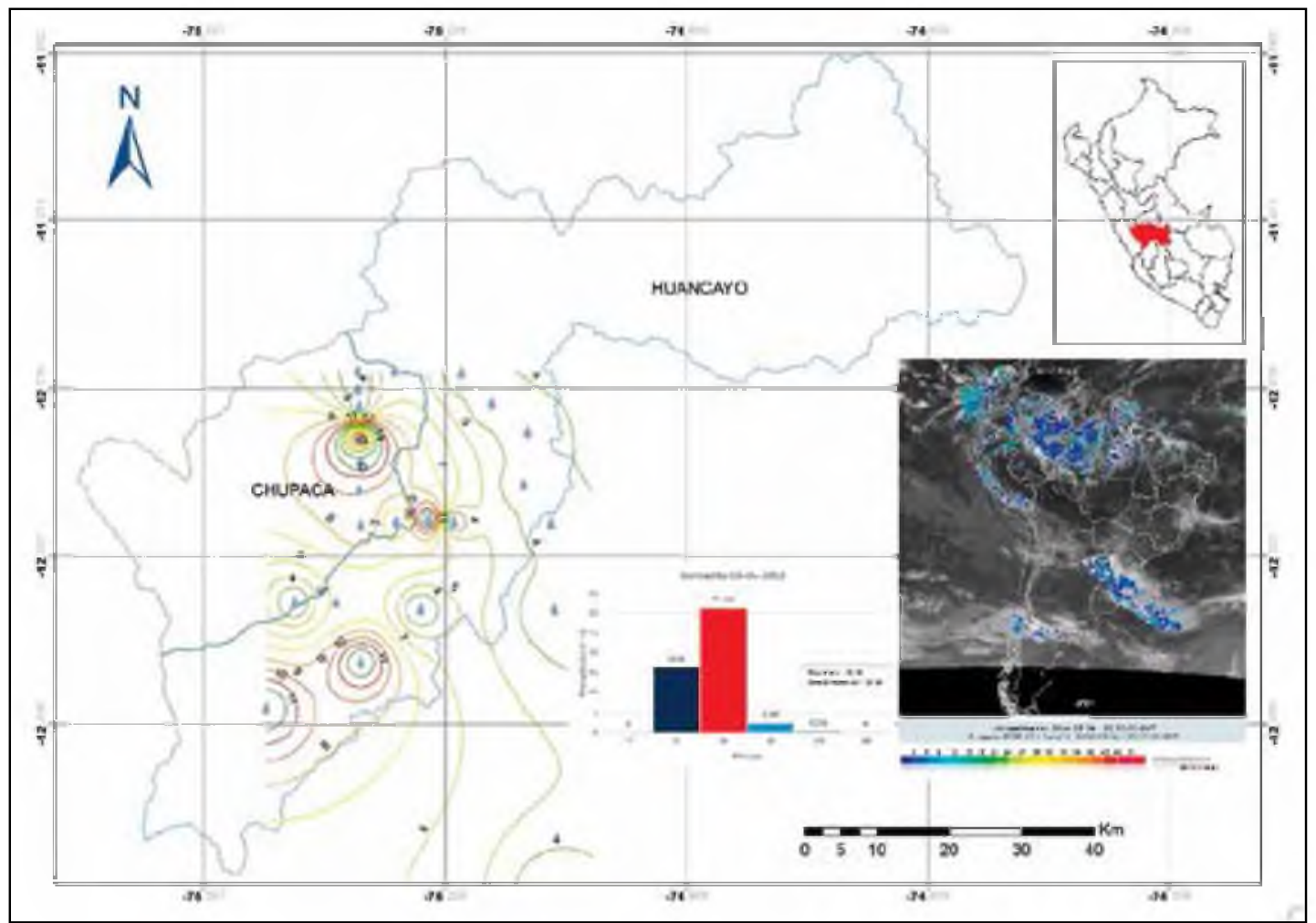

Figura $N^{\circ}$ 7: Tormenta hidrológica del día 4 de mayo de 2014.
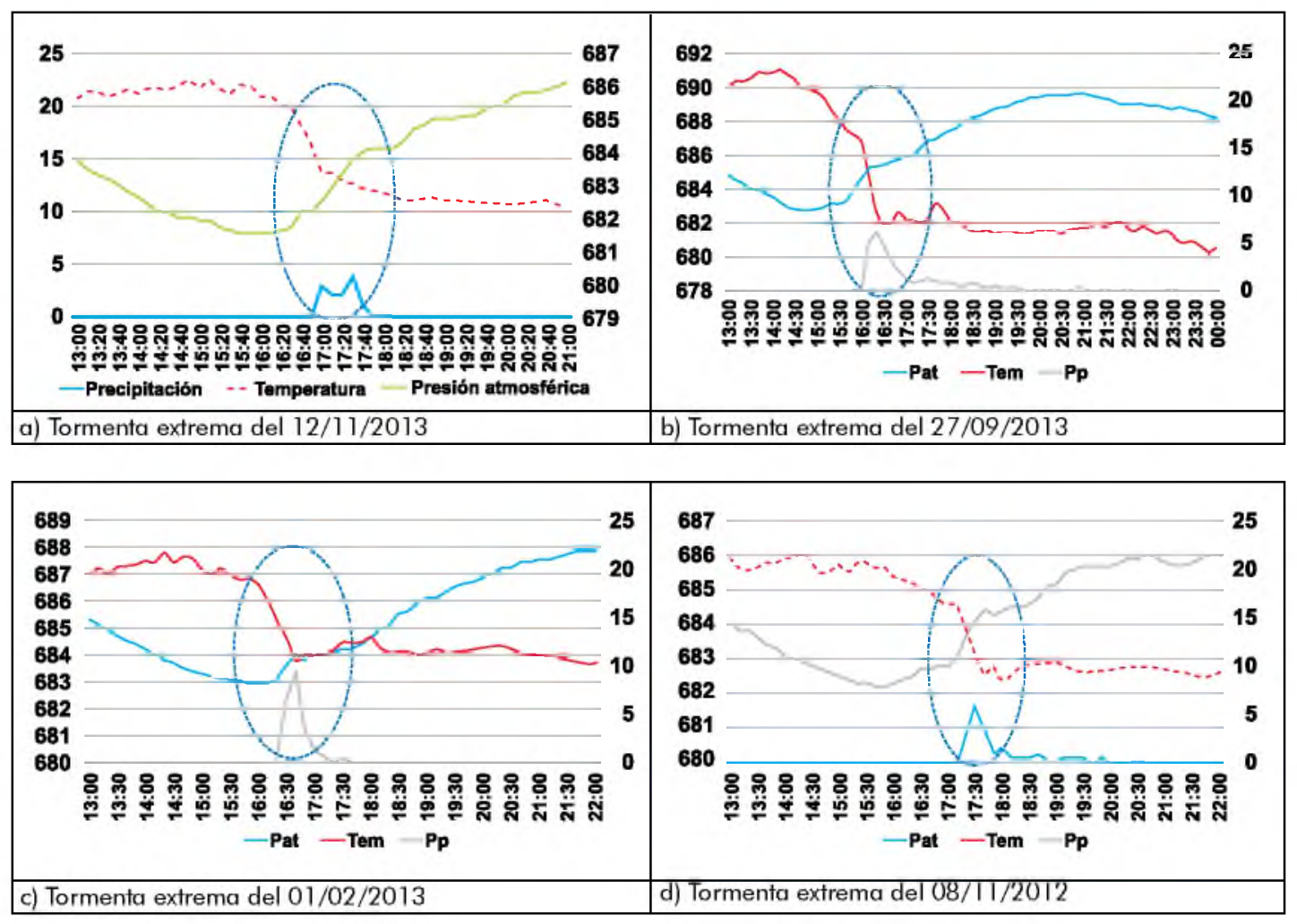

Figura $\mathrm{N}^{\circ} 8$ : Características de las variables en el momento del evento. 


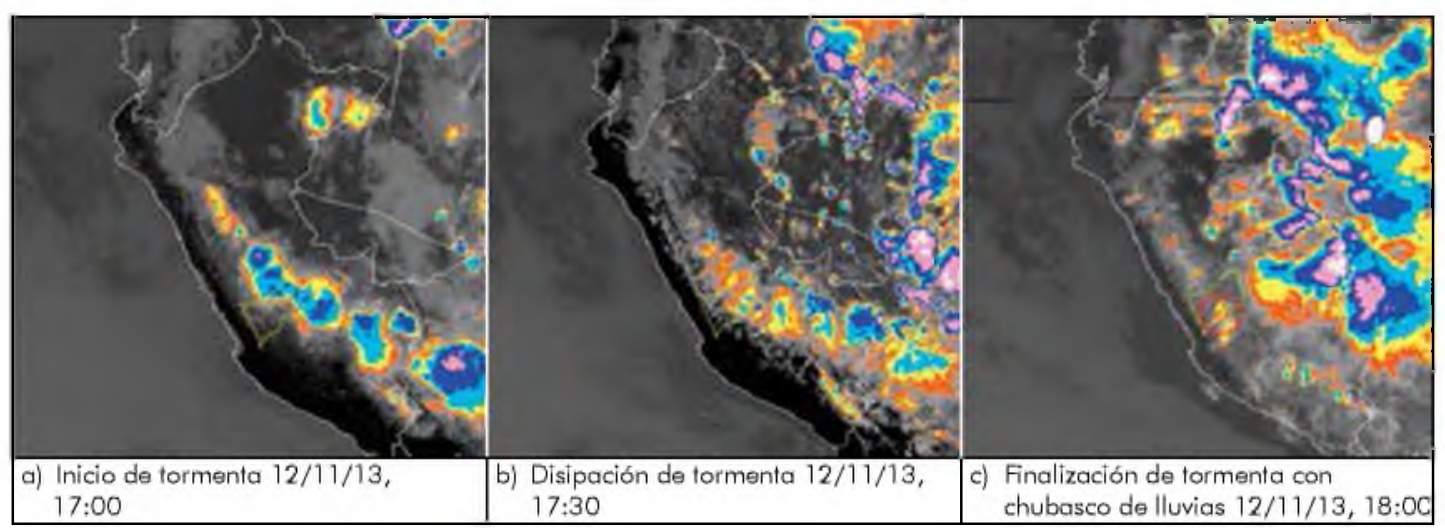

Figura $\mathrm{N}^{\circ}$ 9: Características de frentes de masas de aire para la formación de tormenta moderada.

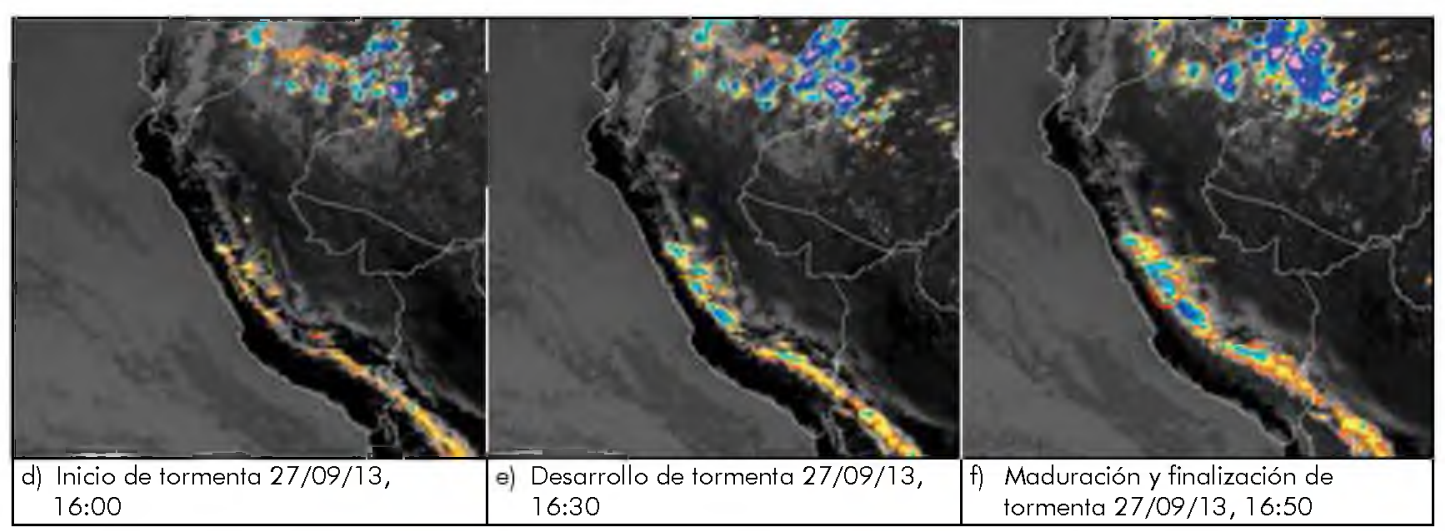

Figura $\mathrm{N}^{\circ}$ 10: Característica de frentes de masa de aire para la formación de tormenta severa de primavera.

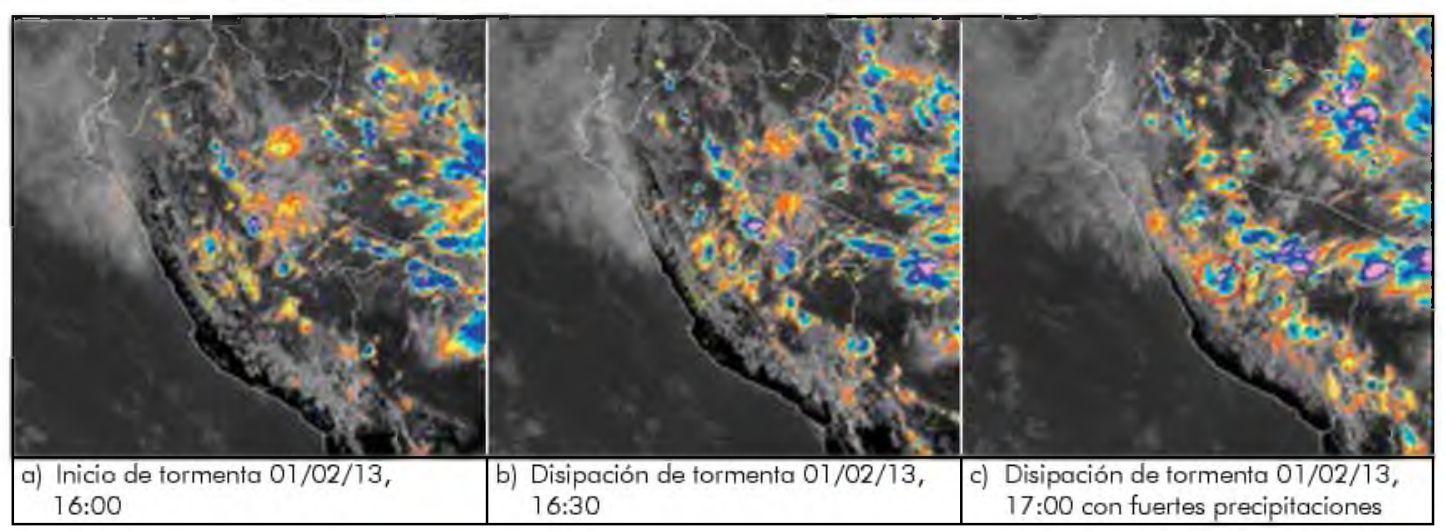

Figura $\mathrm{N}^{\circ} 11$ : Características de frentes de aire para formación de tormentas severas de verano.

que choca con el aire cálido y produce las precipitaciones extremas.

En las figuras 9, 10 y 11 se caracterizan las imágenes satelitales que corresponden a cada evento de tormenta extrema. Inicialmente se observa un calentamiento diferenciado en los bordes de cada masa de aire que se desplaza. Las precipitaciones comunes proceden del encuentro de frentes de aire cálido y frío, en donde la masa frío empuja al cálido y lo desplaza hacia arriba que se enfría formando nubes, para luego generar un movimiento tormentoso que precipita en forma de chubasco o aguacero. Después de producirse las precipitaciones en la imagen se observa una disgregación de las masas de aire. 


\section{DISCUSIÓN}

Las tormentas se presentan como consecuencia de la turbulencia atmosférica, y el encuentro de masas de aire cálido y frío en un momento determinado del tiempo (3). En la zona de estudio se producen aproximadamente 27 tormentas al año entre ligeras y extremas. Estas siempre van acompañados por lluvia, aumento de la velocidad del viento, cambio en las variables de las presiones atmosféricas y el descenso de la temperatura. También en muchos casos se producen truenos y relámpagos especialmente en las tormentas extremas (tabla 2).

Como lo menciona Zamanillo (5), las tormentas presentan una estructura que pueden tener varios centros activos, eso es lo que se observa en las precipitaciones extremas atípicas observadas en el año 2014 (figuras 6 y 7). Estas precipitaciones han sobrepasado los umbrales registrados en una hora, al tener más de $50 \mathrm{~mm}$ en una hora. También lo es en el tiempo, dado que casi nunca debe presentarse en los meses de abril y mayo. Estas características indican el inicio de un nuevo patrón de comportamiento que se asocia al calentamiento global.

En el ciclo de vida de las tormentas registradas en la investigación obedecen a la teoría Bando (7), en donde las depresiones observadas durante la ocurrencia de un evento extremo de tormenta se identifica por la variación de variables importantes de temperatura y presión atmosférica antes del inicio de una gran tormenta (figura 8). Estos indicadores suceden con un aumento inicial de la temperatura y un descenso posterior al momento de las precipitaciones intensas durante la tormenta.

Las precipitaciones moderadas y ligeras se presentaron en los bordes de la ciudad de Chupaca y en la ciudad de Huancayo. Pero las tormentas fuertes todas ellas se concentraron únicamente en la ciudad de Chupaca. Las tormentas extremas se concentraron también en Chupaca y dos en la ciudad de Huancayo (figuras 3, 4 y 5).
Pero debido al proceso descrito por Cueva (3), en dónde indica que son los fenómenos convectivos que producen lluvias intensas por el recalentamiento de las ciudades y los cambios en los parámetros meteorológicos se empiezan a observar horas antes de ocurrir las tormentas, con un cambio negativo en la variación de la temperatura y un aumento de la variable de presión atmosférica (figura 8). En el punto de inflexión de ambas variables ocurren las tormentas más extremas. Pero luego que se originó las precipitaciones la temperatura continúa con una tendencia horizontal, mientras que la presión atmosférica sigue aumentando sus valores.

Para Scofield (14), las precipitaciones de otoño más extremas ocurren por la incidencia de frentes de aire frío al sistema atmosférico y que desplazan al aire cálido de la zona. Estos procesos se pueden verificar al obtener resultados a partir del hidroestimador generados por el satélite GOES-12. La georreferenciación de los puntos y de isoyetas muestra las concentraciones de las intensidades de precipitaciones. La correlación entre los datos del hidroestimador y del pluviómetro tienen una $r=0,75$. Lo que significa que se pueden estimar datos con un nivel de confianza del $95 \%$ a partir de las imágenes satelitales del GOES-12 y georreferenciar (figuras 11 y 12)

Las técnicas propuesta por Campos (10), permiten identificar el comportamiento de la atmósfera y clasificar en cálidas que acumula más agua $y$, por lo tanto, genera más precipitaciones. Pero estas precipitaciones más intensas se originan al inicio de la temporada de primavera o al final de la temporada del otoño, aunque pueden aparecer en cualquier época del año y las condiciones para su formación pueden resumirse en elevada temperatura superficial, elevada humedad atmosférica, inestabilidad de la masa de aire (fuerte gradiente térmico vertical) presencia de bajas presiones y aporte de condiciones de relieve (figuras 9,10 y 11 ). 


\section{Agradecimientos}

Al Instituto Geofísico de Perú por el apoyo científico en la investigación; a la Universidad Continental por el apoyo en la publicación. También a Stream Lab por el apoyo en la sistematización de la información, y a Edsel Arroyo por las descarga de imágenes satelitales.

\section{REFERENCIAS BIBLIOGRÁFICAS}

1. Bluestein H. Synoptic-Dynamic Meteorology in Midlatitudes: Principles of Kinematics and Dynamics Vol 1. Oxford University Press; 1992.

2. Bluestein H. Synoptic-Dynamic Meteorology in Midlatitudes: Observations and Theory of Weather Systems Vol 2. Oxford University Press; 1993.

3. Cuevas E, Rodríguez J. Estadística de las depresiones aisladas en niveles altos. $\checkmark$ Simposio Nacional de Predicción. Madrid: Ministerio de Medio Ambiente. Secretaria General Técnica; 2001.

4. Hernández A. Un estudio de las depresiones aisladas en niveles altos (DANAs) en el sudoeste de Europa basados en mapas isentrópicos de Vorticidad Potencial. IV Simposio Nacional de Predicción. Madrid: Ministerio de Medio Ambiente. Secretaria General Técnica; 1999.

5. Zamanillo E. Tormentas de diseño para la provincia de entre Ríos. 1a ed. Buenos Aires: Univ. Tecnológica Nacional; 2008.

6. David-Novak $H$, Morin $E$, Enzel $Y$. Modern extreme storm and the rainfall thresholds for initiating debris flows on the hyperarid western scapment of the Dead Sea, Israel. Geological Society of America Bulletin. 2004; 116(5-6): 718 728.

7. Bando U, Pereyra D, Natividad M. Curvas intensidad de la lluvia-duraciónperíodo de retorno para tres localidades del estado de Quintana Roo. XII Congreso Nacional de Meteorología.
Cancún; 2002.

8. Takahashi K. Escenarios dimáticos en la Cuenca del río Mantaro. Eventos Meteorológicos Extremos (sequías, Heladas y lluvias intensas) en el Valle del Mantaro. 2012; 1: 79-83.

9. Bell F. Generalized rainfall durationfrequency relationships, J Hydraul Div. 1969; 95: 311-327.

10. Campos D. Procesos del ciclo hidrológico. San Luis Potosí: Editorial Universitaria Potosina, San Luis Potosí; 1987.

11. Campos D, Gómez R. Procedimiento para obtener curvas ID- T a partir de registros pluviométricos. Ingeniería Hidráulica en México. 1990; 5(2): 39 52.

12. Campos D. Procesos del Ciclo Hidrológico. $3^{\underline{a}}$ ed. San Luis Potosí: Editorial Universitaria Potosina; 1998.

13. Chen C. Rainfall intensity-duration frequency formulas. Journal of Hydraulic Engineering. 1983; 109(12): 1603-1621.

14. Scofield R. Comments on "A quantitative assessment of the NESDIS AutoEstimador". Wea. Forecasting. 2001; 16(2): 277- 278.

15. Vicente $G$, Scofield $M$, yMenzel $W$. The operational GOES infrared rainfall estimation technique. Bull. Amer. Meteor. Soc. 1998; 79(9): 1883-1898.

16. Vicente G, Davenport J, Scofield R. The role of orographic and parallax corrections on real time high resolution satellite estimation. Int. J. Remote Sens. 2002; 23(2): $221-230$.

17. Chow V, Maidment D, Mays L. Hidrología Aplicada. Colombia: McGraw Hill; 1994.

18. Froehlich D. Shout-duration-rainfall intensity equations for drainage design, Journal Irigation and Drainage Engineering. 1993; 119(5): 814-828.

19. Froehlich D. Intermediatedurationrainfall intensity equations, Journal of Hydraulic Engineering. 1995; 121(10): 751-756.

20. Genovez A, Pegogaro R. Análisis y Evaluación de Ecuaciones de Lluvia Intensa Generalizada sugeridas por el CPTEC, Ingeniería Hidráulica en 
México. 2001; 16(3): 15-25.

21. Haber A, Runyon R. Estadística General. Bogotá: Fondo Educativo Interamericano; 1973.

22. Scofield R. The NESDIS operational convective precipitation technique. Mon Wea Rev. 2008; 115: 1773-1792. 